\section{Management von Streptokokken der Serogruppe A}

$\mathrm{K}$ linische Symptome wie Fieber und Rötungen im Rachen sind keine verlässlichen Indikatoren für Infektionen mit Streptokokken der Gruppe A (GAS). Scoring-Systeme wie der McIssaacScore sind bei Erwachsenen validiert, waren in Studien mit Kindern aber nicht unbedingt aussagekräftig, erläuterte Prof. Dr. Pierre Smeesters aus Brüssel. Schnelltests können GAS-Infektionen effizient nachweisen und müssen nicht durch weitere Bakterienkulturen bestätigt werden. Man muss jedoch im Hinterkopf behalten, dass etwa $10 \%$ der Kinder GAS-Träger sein können. Da Komplikationen eher rar sind, und die „number to treat“ mit 200 sehr hoch ist, empfehlen Leitlinien in Europa und in
Australien bei GAS meist keine Routinegabe von Antibiotika, während in den USA eine Routinegabe nahegelegt wird.

Invasive GAS können zu seltenen, aber schweren Komplikationen führen: Bei einem an Windpocken erkrankten fiebrigen kleinen Mädchen hatte sich in einem heftig geröteten und geschwollenen Arm eine nekrotisierende Fasziitis entwickelt, die umgehend operativ behandelt werden musste. In der Blutkultur wurden danach multisensitive GAS nachgewiesen. Trotz des dramatischen Eingriffs sei hier die Gabe von Penicilllin ausreichend, betonte Smeesters. Bisher wurden noch nie GAS mit Penicillin-Resistenzen beschrieben. Clindamycin könnte wegen eines anderen
Angriffspunktes in der bakteriellen Proteinsynthese ergänzend eingesetzt werden, aber Penicillin nicht ersetzen. Diese Kombination zeigte in Studien eine geringere Mortalität bei schweren GASKomplikationen, was auch bei intravenösen Immunglobulinen der Fall war.

Analog zu Infektionen mit Neisseria meningitidis wird bei schweren Fällen von GAS - mit höherer Mortalität als bei Neisserien - auch die prophylaktische Antibiotikagabe an Haushaltsangehörige erwogen. Daten zeigen jedoch laut Smeesters, dass die Prophylaxe nur bei einer Exposition von mehr als 24 Stunden pro Woche und nur in der nahen Umgebung wie der Familie sinnvoll ist.

Martina Freyer

Smeesters P. Group A strep diagnosis and management

\title{
Leishmaniose in Europa: Hunde besser nicht streicheln
}

nfektiologen aus der klinischen Praxis beschreiben die Falldiskussionen in Brighton als ihr persönliches ESPIDHighlight, in der die diagnostische Expertise der Zuhörer herausgefordert wird. Dr. Jolanta Bernantoniene von der Universität Bristol erläuterte, dass hinter den bei Kleinkindern häufigen fiebrigen Symptomen eine klassische Tropenkrankheit stecken kann. Ein 12 Monate altes Mädchen litt seit etwa 10 Wochen

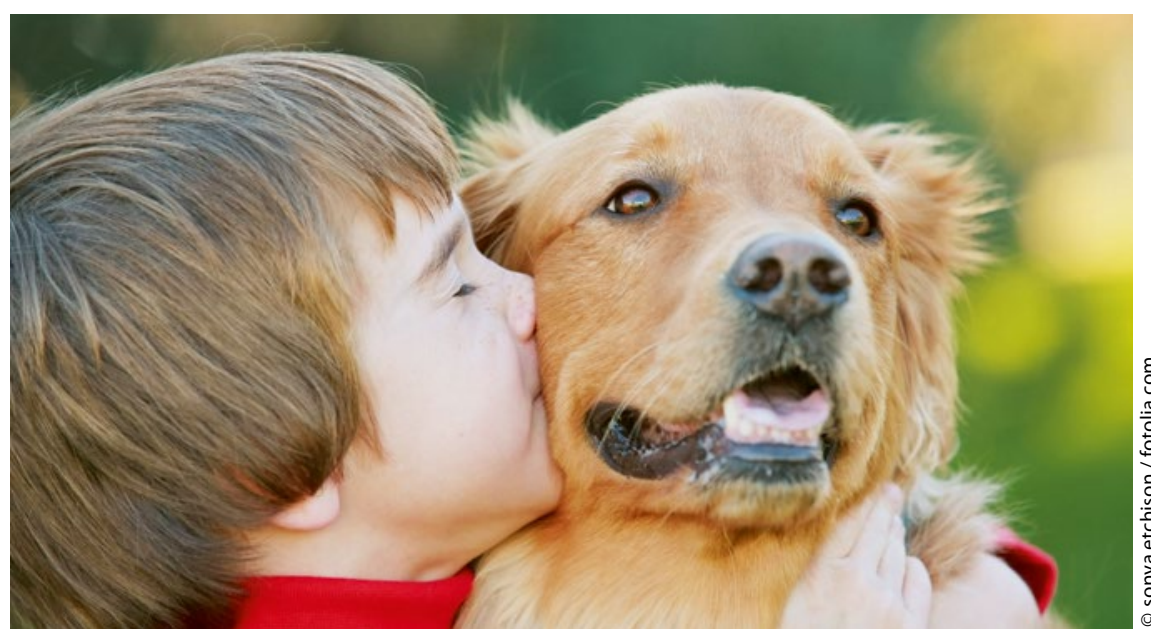

an Fieber, Erkältungen, Husten und Konjunktivitis. Das Kind war nicht geimpft, die Familie lebt auf einem Boot, die Eltern sind Anhänger der Homöopathie. Mit zunehmender Symptomatik landete das Mädchen mit Hepatosplenomegalie in der Klinik. Nach gescheiterten Therapieansätzen mit Antibiotika und Eisengabe brachte die Anamnese eine Portugal-Reise der Familie zutage: Das kleine Mädchen hatte sich wohl

beim Spielen an Land im Kontakt mit infizierten Hunden mit Leishmanien angesteckt.

Leishmanien, die durch Sandfliegen übertragen werden, kommen im gesamten Mittelmeerraum vor. Im Landesinneren Portugals fungieren Hunde als Reservoir für Leishmania infantum. Mit der Ausbreitung der Sandfliege in nördliche Gefilde ist auch in Mitteleuropa häufiger mit Leishmanien zu rechnen.

Typisch für die viszerale Leishmaniose sind laut Bernantoniene nicht nur befallene Lymphknoten, sondern auch multiple Läsionen in der Milz sowie Makrophagen, die Erythrozyten enthalten. Bei erkrankten Kindern sollten wegen der ähnlichen Symptome eine hämophagozytische Lymphohistiozytose ausgeschlossen werden. Die auch als DumDum-Fieber, Schwarzes Fieber oder Kala-Azar bezeichnete Infektion mit den intrazellulären protozoischen Parasiten konnte bei dem kleinen Mädchen medikamentös behandelt werden. Gerade bei Kleinkindern, die Kontakt mit Hunden suchen, sollte man an diese ProtozoenErkrankung denken.

Martina Freyer

Bernantoniene J. Interactive case session 